\title{
Effects of convection-enhanced delivery of bevacizumab on survival of glioma-bearing animals
}

\author{
Weijun Wang, MD, ${ }^{1}$ Walavan Sivakumar, MD, ${ }^{1}$ Shering Torres, BS, ${ }^{2}$ Niyati Jhaveri, MS, ${ }^{2}$ \\ Vijaya Pooja Vaikari, MS, ${ }^{2}$ Alex Gong, BA, ${ }^{1}$ Adam Howard, BS, ${ }^{2}$ Encouse B. Golden, MD, \\ Stan G. Louie, PharmD, PhD, ${ }^{4}$ Axel H. Schönthal, PhD, ${ }^{3}$ Florence M. Hofman, PhD, ${ }^{2}$ and \\ Thomas C. Chen, MD, PhD'
}

Departments of ${ }^{1}$ Neurosurgery, ${ }^{2}$ Pathology, and ${ }^{3}$ Molecular Microbiology and Immunology, Keck School of Medicine; and ${ }^{4}$ Department of Clinical Pharmacy and Pharmaceutical Economics and Policy, School of Pharmacy, University of Southern California, Los Angeles, California

\begin{abstract}
OBJECT Bevacizumab (Avastin), an antibody to vascular endothelial growth factor (VEGF), alone or in combination with irinotecan (Camptosar [CPT-11]), is a promising treatment for recurrent glioblastoma. However, the intravenous (IV) administration of bevacizumab produces a number of systemic side effects, and the increase in survival it provides for patients with recurrent glioblastoma is still only a few months. Because bevacizumab is an antibody against VEGF, which is secreted into the extracellular milieu by glioma cells, the authors hypothesized that direct chronic intratumoral delivery techniques (i.e., convection-enhanced delivery [CED]) can be more effective than IV administration. To test this hypothesis, the authors compared outcomes for these routes of bevacizumab application with respect to animal survival, microvessel density (MVD), and inflammatory cell distribution.
\end{abstract}

METHODS Two human glioma cell lines, U87 and U251, were used as sources of intracranial tumor cells. The glioma cell lines were implanted into the brains of mice in an orthotopic xenograft mouse tumor model. After 7 days, the mice were treated with one of the following: 1) vehicle, 2) CED bevacizumab, 3) IV bevacizumab, 4) intraperitoneal (IP) irinotecan, 5) CED bevacizumab plus IP irinotecan, or 6) IV bevacizumab plus IP irinotecan. Alzet micro-osmotic pumps were used to introduce bevacizumab directly into the tumor. Survival was monitored. Excised tumor tissue samples were immunostained to measure MVD and inflammatory cell and growth factor levels.

RESULTS The results demonstrate that mice treated with CED of bevacizumab alone or in combination with irinotecan survived longer than those treated systemically; CED-treated animals survived $30 \%$ longer than IV-treated animals. In combination studies, CED bevacizumab plus CPT- 11 increased survival by more than $90 \%$, whereas IV bevacizumab plus CPT-11 increased survival by $40 \%$. Furthermore, CED bevacizumab-treated tissues exhibited decreased MVD compared with that of IV-treated tissues. In additional studies, the infiltration of macrophages and dendritic cells into CED-treated animals were increased compared with those in IV-treated animals, suggesting a highly active inflammatory response taking place in CED-treated mice.

CONCLUSIONS The administration of bevacizumab via CED increases survival over that of treatment with IV bevacizumab. Thus, CED of bevacizumab alone or in combination with chemotherapy can be an effective protocol for treating gliomas.

http://thejns.org/doi/abs/10.3171/2015.1.FOCUS14743

KEY WORDS glioma; bevacizumab; convection-enhanced delivery; monotherapy; combination therapy

$\mathrm{V}$ ASCULAR endothelial growth factor (VEGF) is a key regulator of tumor growth and angiogenesis. ${ }^{8,10}$ This growth factor is responsible for endothelial cell proliferation, migration, survival, and the recruitment of endothelial cell progenitor cells from bone marrow to the tumor site. ${ }^{29}$ Tumor cells, as well as cancer stem cells and infiltrating macrophages, secrete VEGF., $2,911,29$

Bevacizumab (Avastin), a humanized monoclonal antibody to VEGF, was the first drug to be developed as an angiogenesis inhibitor and was approved by the US Food

ABBREVIATIONS CED = convection-enhanced delivery; IP = intraperitoneal; IT = intratumoral; IV = intravenous; MVD = microvessel density; VEGF = vascular endothelial growth factor; VEGFR = VEGF receptor.

SUBMITTED October 30, 2014. ACCEPTED January 6, 2015

INCLUDE WHEN CITING DOI: 10.3171/2015.1.FOCUS14743.

DISCLOSURE Dr. Chen reports owning stock in Pharmaco-Kinesis Corp. The following sources funded this study: grants from the Sounder and Kriegel Foundations (to T.C.C.) and Pharmaco-Kinesis Corp. (to F.M.H.). 
and Drug Administration for the treatment of glioma in conjunction with other chemotherapeutic agents, particularly irinotecan and carboplatinum.,21,30 Bevacizumab treatment has been shown to have a wide range of effects based on its binding to soluble VEGF, thus reducing the activation of the VEGF signaling pathway. ${ }^{10}$ During Phase I clinical trials in patients with rectal carcinoma, treatment with bevacizumab in combination with chemotherapy resulted in a significant reduction in tumor microvessel density (MVD) and tumor blood volume. ${ }^{21}$ Bevacizumab has also been shown to have some degree of success in the treatment of recurrent gliomas. ${ }^{6}$ In newly diagnosed gliomas, however, bevacizumab produced a significant improvement in progression-free survival but no significant improvement in overall survival compared with that of historic controls. ${ }^{24}$ Thus, systemic administration of bevacizumab alone or in combination with irinotecan has exhibited limited effects on glioma progression without significant overall survival improvement in recurrent and newly diagnosed tumors. ${ }^{12,19}$

We hypothesize that convection-enhanced delivery (CED) of bevacizumab is more effective than other types of administration because the location of bevacizumab's interaction with VEGF is critical. VEGF is secreted by glioma cells and is present in the interstitial spaces of the tumor. It interacts with endothelial cells via VEGF receptors (VEGFRs) on the luminal side of the blood vessel, which causes the blood vessels to dilate and opens up the tumor-blood interface. ${ }^{16}$ Intravenous (IV) bevacizumab works by preventing VEGF from binding to the VEGFR on the endothelial cells. The question is, does bevacizumab work more effectively to bind to and eliminate the VEGF secreted by glioma cells when it is delivered into the interstitial space?

Moreover, although bevacizumab has been successful in initially inhibiting blood vessel growth and endothelial cell proliferation, continued long-term exposure may result in gliomatosis cerebri. ${ }^{17}$ Further complications of the systemic administration of this agent are increased risks of renal toxicity, poor wound healing, deep venous thrombosis, and hypertension. ${ }^{17,19}$

On the basis of these clinical problems that potentially result from systemic administration, we tested whether chronic administration of bevacizumab intratumorally (IT) (i.e., CED) has beneficial effects in an in vivo xenograft model. The hypothesis of this study is that CED of bevacizumab is more effective than IV administration for slowing tumor progression and for animal survival.

\section{Methods}

\section{Cell Culture and Therapeutic Drugs}

Human glioma cells (U87 or U251) were cultured in Dulbecco's modified Eagle's medium supplemented with $10 \%$ fetal bovine serum and $1 \%$ penicillin-streptomycin and incubated in 5\% $\mathrm{CO}_{2}$. Bevacizumab (Avastin) (Genentech, Inc.) and irinotecan (Camptosar [CPT-11], Pharmacia Union) were purchased from the USC/Norris Comprehensive Cancer Center pharmacy. Bevacizumab and irinotecan have been approved by the US Food and Drug Administration for clinical use.
A 28-day sterile Alzet micro-osmotic pump (model 1004) was used in these experiments. The Alzet pump has a reservoir volume of $90 \mu \mathrm{l}$ and a delivery rate of 0.11 $\mu \mathrm{l} /$ hour. The brain infusion kit, which includes the pump and distribution tubing, was purchased from the Durect Corporation.

\section{In Vivo Procedure for Tumor Cell Implantations}

The animal protocol was approved by the institutional animal care and use committee of the University of Southern California. All the mice were maintained in a pathogen-free environment throughout the experiment. Briefly, each athymic nu/nu mouse (Harlan, Inc.) was anesthetized with $10 \mathrm{mg} / \mathrm{kg}$ ketamine-xylazine and fixed into a stereotactic head frame (Harvard Apparatus). A 1.5-mm bur hole was drilled $1 \mathrm{~mm}$ anterior to the coronal suture on the right hemisphere and $2 \mathrm{~mm}$ lateral from the midline. A Hamilton syringe fixed onto the head frame was used to inject human glioma cells $\left(2 \times 10^{5} / 5 \mu \mathrm{l}\right)$ into the right frontal lobe of the brain. The skin incision was then closed with 4-0 silk thread. Appropriate medications were provided to reduce pain.

\section{In Vivo Procedure for Alzet Micro-Osmotic Pump Implantations}

Seven days after the tumor cell implantations, an Alzet pump was implanted into each mouse. Before implantation of the pump, the Alzet micro-osmotic pump was filled with the desired dose of reagent. In addition, a plastic tube primed with the agent was used to connect the brain infusion kit (Durect Corporation) to the brain. The assembled delivery system was stored at $4^{\circ} \mathrm{C}$ overnight before implantation. Bevacizumab was delivered into the tumor using chronic pump-mediated delivery, defined as "convection-enhanced delivery" or CED. This CED method was used because it has the advantage of achieving the desirable drug concentration in the microenvironment of the glioma while avoiding the use of high initial doses.

To anchor a pump to each animal, an incision was made in the scalp along the previous surgical scar. The caudal edge of the incision was pulled, the connective tissues were separated, and a subcutaneous tunnel was prepared. The Alzet micro-osmotic pump was pushed backward to adjust the location of the brain infusion kit directly above the hole in the skull previously drilled for tumor cell implantation. The infusion kit (plastic tubing) was inserted gently into the formed tumor. The plastic platform end was fixed onto the skull using instant super glue. The area around the skin incision was sterilized and sutured with 4-0 silk thread. The contents of the Alzet microosmotic pump were released into the tumor at a rate of $0.11 \mu \mathrm{l}$ per hour. The treatment given to the animals $(\mathrm{n}=$ 5) was as follows: 1) vehicle (IgG), 2) IV bevacizumab, 3) CED bevacizumab, 4) irinotecan, 5) IV bevacizumab plus irinotecan; or 6) CED bevacizumab plus irinotecan. IV injections were given through the tail-vein route. The irinotecan was always administered intraperitoneally (IP) at a dose of $12 \mathrm{mg} / \mathrm{m}^{2}$ or $4 \mathrm{mg} / \mathrm{kg}$ (in saline) on Days 1-5 and 8-12. Equivalent doses of bevacizumab were either administered by IV injection at $10 \mathrm{mg} / \mathrm{kg}$ once every 14 days ( 2 injections in 28 days) or delivered continuously 
by direct CED infusion (Alzet micro-osmotic pump) for 28 days, resulting in a total dose of bevacizumab of 0.8 mg. After treatment was completed (28 days), the animals were maintained without any treatment until they died spontaneously or were killed because of the tumor burden. Animals treated with vehicle received CED of saline using the Alzet micro-osmotic pump as described above.

\section{Immunostaining}

Frozen mouse brain tumor tissues were sectioned at 8 $\mu \mathrm{m}$, fixed in acetone, washed in phosphate-buffered saline, and blocked with Sea Block (Thermo Scientific). The sections were stained with anti-CD11c or F4/80 (Abcam). Subsequently, the tissues were incubated with corresponding secondary antibodies (Vector Laboratories) for 45 minutes, incubated with avidin-biotin peroxidase complex (ABC kit; Vector Laboratories) for 30 minutes, and treated with amino-ethylcarbazol substrate for 10 minutes. The samples were counterstained with hematoxylin for 1 minute; a red precipitate indicated positive staining. We included no primary antibody or irrelevant primary antibody for controls. Evaluations for macrophages (F4/80) and dendritic cells (CD11c) were performed at $\times 200$ magnification. The cells were quantified using ImageJ software.

\section{MVD Analysis}

Frozen mouse brain tissues were treated as described above using anti-CD31 (PECAM-1) (BD Pharmingen). MVD data were obtained by evaluating 10-20 random fields per tumor at $\times 200$ magnification. Tissues from at least two mice per group were analyzed. MVD was quantified using ImageJ software.

\section{Statistical Analysis}

Survival data were plotted using the Kaplan-Meier method. Survival time was calculated from the date of tumor injection to the date of death. The log-rank test was used to determine statistical significance; a $p$ value of $<$ 0.05 was considered significant.

\section{Results}

\section{Intracerebral Infusion of Bevacizumab Did Not Result in Intraparenchymal Hemorrhages in Normal Brains}

To test the safety of intracerebral administration of bevacizumab, the agent was administered directly into the brain. Briefly, $0.8 \mathrm{mg}(200 \mu \mathrm{l})$ of bevacizumab, equivalent to $10 \mathrm{mg} / \mathrm{kg}$ one time per 14 days, was injected IV into the striatum of the frontal lobe in normal mouse brains ( $\mathrm{n}=$ 12). As shown in six representative brain-specimen crosssections (Fig. 1), this procedure caused no visible evidence of intracerebral hemorrhage.

\section{CED of Bevacizumab Increased Survival of Glioma-Bearing Mice}

To determine whether there were any clinical advantages to delivering bevacizumab locally, intracranial tumorbearing mice were given this agent through either CED or the IV route as a monotherapy or in combination with the chemotherapeutic agent, irinotecan (CPT-11). The chemotherapeutic agent was administered via IP route at $4 \mathrm{mg}$ / $\mathrm{kg}$. Two different glioma cell lines were used to provide evidence for a more general response. In U251 tumor-bearing animals, the results showed that CED significantly ( $\mathrm{p}$ $<0.002$ ) increased survival (Fig. 2A) over that of vehicletreated (immunoglobulin G) controls; CED of bevacizumab-treated animals survived 30\% (10 days) longer than the controls, whereas there was no significant difference between the control and IV treatments. CPT-11 as a monotherapy was not effective. CPT-11 in combination with bevacizumab enhanced survival of the CED- and IV-treated
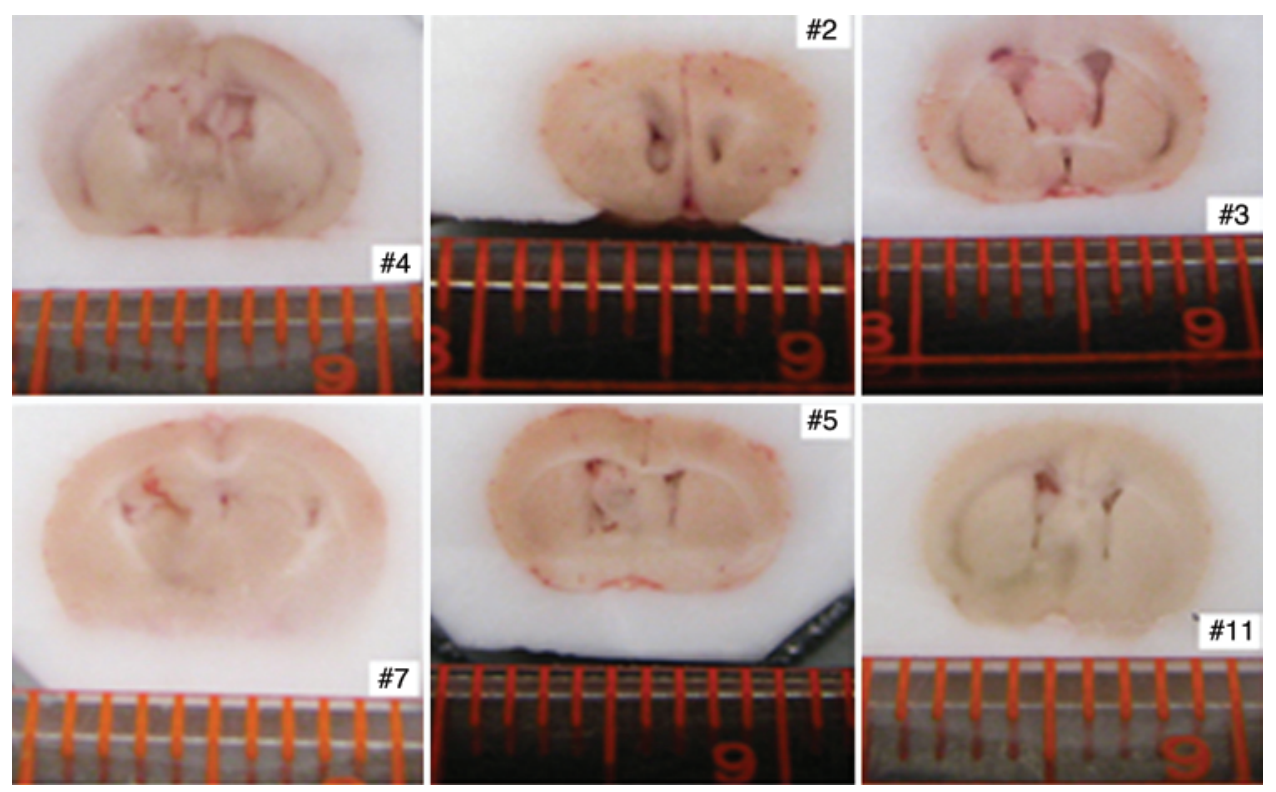

\#5

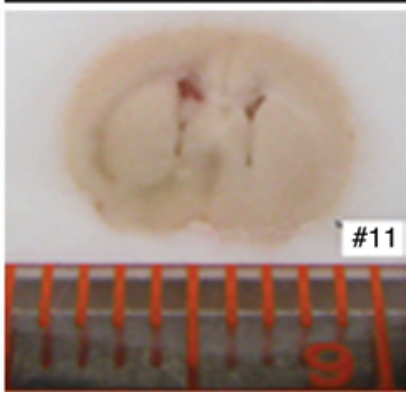

FIG. 1. Intracerebral infusion of bevacizumab resulted in no hemorrhaging. Bevacizumab $(0.8 \mathrm{mg})$ was injected into the striatum of the frontal lobe in normal mouse brains $(n=12)$. Cross-sections of the brains reveal no evidence of intracerebral hemorrhage. The photos are of 6 representative brains. 
animals ( $\mathrm{p}<0.02)$; however, treatment with CED of bevacizumab combined with CPT-11 prolonged survival longer than IV administration of bevacizumab with CPT-11 ( $\mathrm{p}<$ 0.002 ).

In a second experiment using the human glioma cell line U87 (Fig. 2B), the results revealed that the combination therapy of CPT-11 with CED of bevacizumab nearly doubled the survival times of mice treated with IV bevacizumab and CPT-11. These data confirm that CED of bevacizumab provided a significant survival benefit for the treatment of brain tumors for both monotherapy and combination therapies.

\section{CED of Bevacizumab Decreased Tumor MVD}

On the basis of in vivo data indicating that CED of bevacizumab, either alone or in combination with irinotecan, increased animal survival compared with that of systemic (IV) administration, we analyzed tumor tissue samples to determine the potential mechanism(s) responsible for this improved survival. To accomplish this goal, tumor tissues were obtained from animals at the time of death
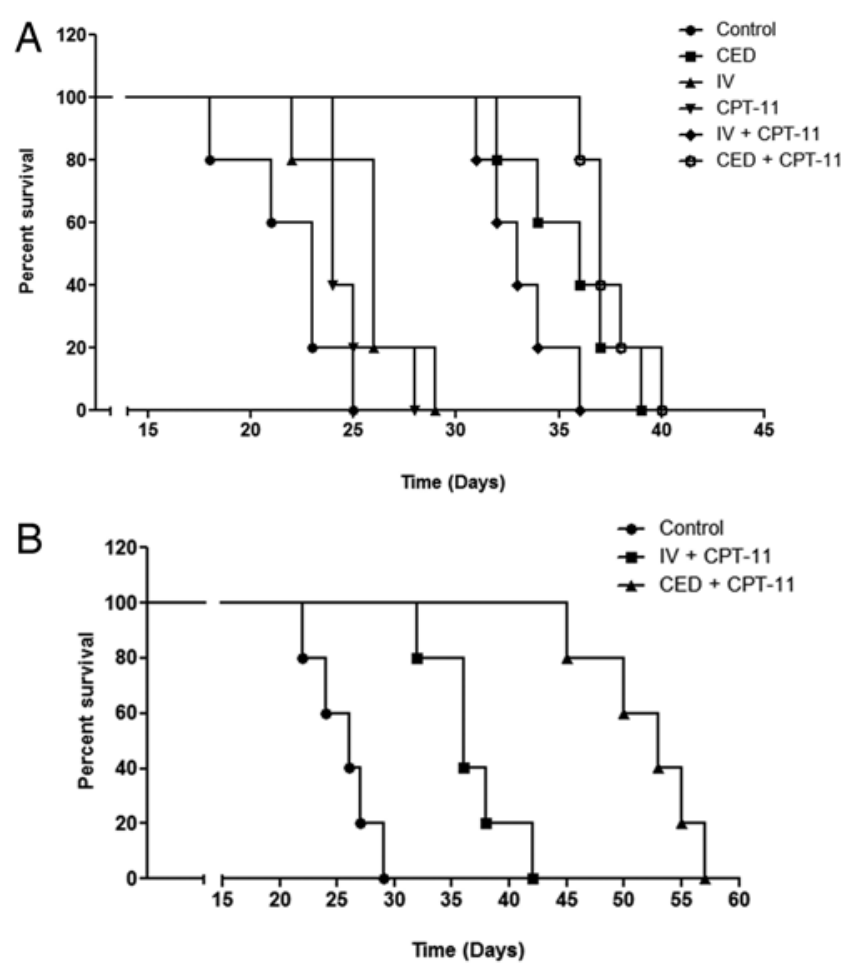

FIG. 2. CED of bevacizumab enhanced survival. A: Animals bearing U251 tumors were given vehicle control with immunoglobulin G, irinotecan (CPT-11), IV bevacizumab, or CED bevacizumab as a monotherapy or IV bevacizumab plus irinotecan or CED bevacizumab plus irinotecan as a combination therapy. Kaplan-Meier analysis shows that CED of bevacizumab significantly increased survival $(p<0.02)$ compared with IV administration when the agent was administered alone. Combination therapy of CED bevacizumab and irinotecan was also significantly more effective than IV bevacizumab and irinotecan $(p<0.002)$. B: Animals bearing U87 tumors were given vehicle control or IV bevacizumab plus irinotecan or CED bevacizumab plus irinotecan as a combination therapy. The Kaplan-Meier analysis shows that combination therapy with CED of bevacizumab and irinotecan was significantly more effective than IV bevacizumab and irinotecan $(p<0.002)$. and analyzed. Because bevacizumab is an antiangiogenic agent, we stained the tumor tissues with anti-CD31, a marker for endothelial cells, and quantified the MVD. The staining results (Fig. 3A) showed a marked reduction in the number of blood vessels in the CED-treated animals compared with those in the IV-treated animals. MVD was calculated (Fig. 3B), and the data show that CED of bevacizumab significantly reduced MVD $(\mathrm{p}<0.01)$ compared with that in the IV group. CPT-11 alone did not have significant effects on MVD. However, combination therapy with either CED of bevacizumab or IV bevacizumab plus CPT-11 significantly decreased MVD ( $<<0.0001)$. The results in the U87 human glioma cell line were similar; CED of bevacizumab was more effective than IV bevacizumab in reducing MVD (data not shown). Chemotherapy in conjunction with bevacizumab caused decreased MVD, which was reflected in the survival.

\section{CED of Bevacizumab Increased Accumulation of Inflammatory Cells in Tumor Tissues}

Inflammatory cells have been shown to play an important role in regulating the host's immune response to a tumor. ${ }^{14}$ The proinflammatory responses initiated by macrophages and dendritic cells are key to blocking tumor progression. To test whether there are differences in the inflammatory response as a result of the site of drug administration, tissues were analyzed for the presence of macrophages and dendritic cells. The red precipitate indicates positive staining. Our results show that macrophages were distributed throughout the tumor tissues in all the groups (Fig. 4A); however, the number of positive cells in the CED bevacizumab plus CPT-11 cohort was significantly greater than that in the IV bevacizumab plus CPT-11 group ( $<$ 0.002) (Fig. 4B). Specimens from the vehicle-treated mice did not show this increased macrophage accumulation. Dendritic cells, often associated with macrophages, function as effective antigen-presenting cells. ${ }^{23}$ Tumor tissues were stained for dendritic cells using CD11c (Fig. 5A). The results show increased expression of dendritic cells in the group treated with a combination of CPT-11 and CED of bevacizumab compared with that in the group treated with CPT-11 and IV bevacizumab; however, these differences were not significant $(p=0.064)$ (Fig. 5B). It should be noted that CD11c can also identify a subpopulation of macrophages. ${ }^{34}$ These data indicate that an intense inflammatory response may be taking place in the tumors treated with CED bevacizumab plus CPT-11, which suggests that the inflammatory response may contribute to a delay in tumor progression.

\section{Discussion}

In this study, we addressed the question of whether there were differences in survival between animals treated with bevacizumab administered by CED or systemically (IV) and how effective bevacizumab as a monotherapy was compared with combination therapy with irinotecan. The results demonstrate that bevacizumab, when given via CED, is more effective than IV administration in increasing survival. Furthermore, in combination studies, CED of bevacizumab together with the chemotherapeutic agent 


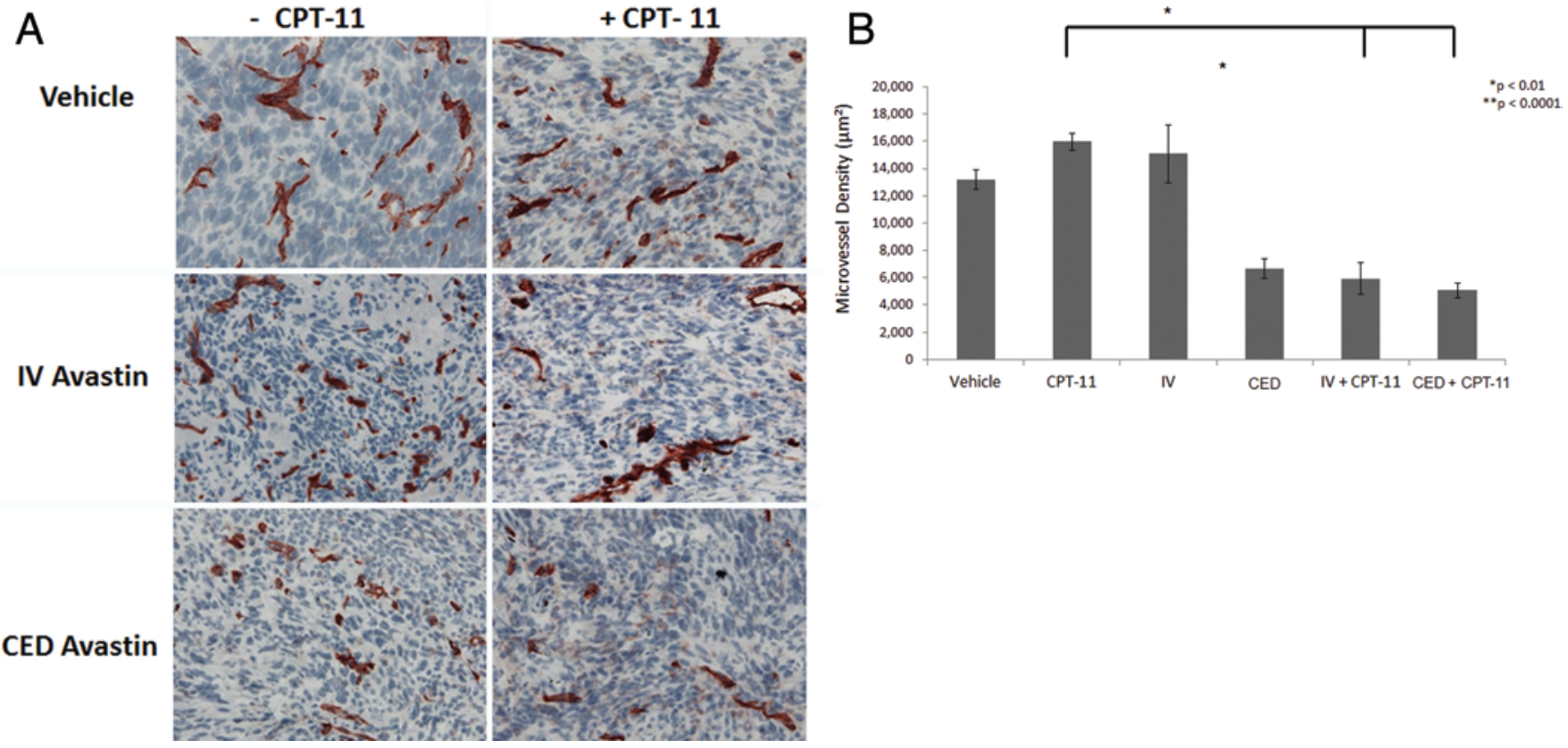

FIG. 3. Bevacizumab treatment decreased MVD. Tumor tissues from the different animal groups were immunostained for blood vessels using anti-CD31. A: Histological specimens show fewer blood vessels in CED bevacizumab-treated tumors than in IV bevacizumab-treated specimens. Red precipitation indicates positive staining. Original magnification $\times 200$. B: MVD analysis shows that animals that received CED of bevacizumab had significantly fewer blood vessels $(p<0.001)$ than those that received IV bevacizumab. Vessels were analyzed using ImageJ.

irinotecan was the most effective for survival. We investigated the potential mechanism of this delay in tumor growth.

The rationale for using antiangiogenic therapy for gliomas is apparent because of the highly vascular nature of these tumors. Clinical trials of bevacizumab found that, as a monotherapy, bevacizumab showed improved progression-free survival in newly diagnosed gliomas. ${ }^{31}$ However, no significant overall increase in survival was reported. ${ }^{12,31}$

VEGF, also known as vascular permeability factor, is critical for the maintenance of tight junctions in the bloodbrain barrier. ${ }^{18}$ Gliomas produce high levels of VEGF, which results in increased vascular permeability and subsequent perivascular edema; bevacizumab therapy reduces vascular permeability. Reduced permeability causes tumors to go from gadolinium enhancing to nonenhancing and thereby appear to shrink. Tightening the blood-tumor interface with bevacizumab results in a reduction in steroid dose and dependency on a long-term basis. ${ }^{19}$ Clinical observations have suggested that reduced brain edema in these patients adds significantly to disease-free survival. ${ }^{31}$ Thus, bevacizumab functions to decrease edema and increase vascular regression.

Recent evidence ${ }^{18}$ has suggested that the effects of bevacizumab treatment may be a result of the "normalization" of blood vessels, as defined by changes in endothelial cell morphology and reduction in vascular permeability. ${ }^{18}$ The hypothesis is that the positive effects of this normalization of blood vessels enable increased blood flow in the tumor vessels and thereby enhance the distribution of chemotherapy. In our studies, we observed that CED of bevacizumab caused enlarged, dilated blood vessels (data not shown), which also may enable more chemotherapeu- tic agents to enter into the tumor in a manner other than normalization. Bevacizumab may have a direct therapeutic effect on tumor vasculature; we show here, and others have reported, that bevacizumab can be effective as a monotherapy, ${ }^{4,9,31}$ which suggests that the effects of bevacizumab can be independent of chemotherapy. However, because combination therapy with bevacizumab is more effective than monotherapy, the normalization of vessels is important but only partly responsible for the effects of bevacizumab therapy. To obtain the benefits of vascular normalization from bevacizumab, combination therapy with cytotoxic agents would be most useful. There is some controversy over bevacizumab toxicity in glioma cells. Studies have shown that there is no direct effect on glioma cell proliferation. However, at medium and large doses, bevacizumab triggers vascular regression, decreased tumor growth, and prolonged survival. ${ }^{31}$ Recent reports indicated that bevacizumab signals through the Akt and Erk signaling pathways. ${ }^{27,28}$ Recent evidence suggests that glioma stem cells produce VEGF and interact with the vasculature, ${ }^{3}$ which indicates that bevacizumab may regulate the survival of tumor stem cells. Gliomas have been shown to secrete VEGF and express VEGFRs, although these receptors are most prominently expressed on endothelial cells. ${ }^{25}$ Although there is a clear understanding of VEGFR expression on endothelial cells, there is currently no clear consensus on VEGFR expression in tumor tissues. ${ }^{15}$ VEGF acting in a paracrine manner may be responsible for tumor cell survival, migration, and invasion. ${ }^{29}$ Thus, bevacizumab functions directly on the tumors and on tumor vasculature ${ }^{13}$. Therefore, CED of bevacizumab may have a greater effect on gliomas than IV bevacizumab. In this study, the levels of VEGF in the different groups were 


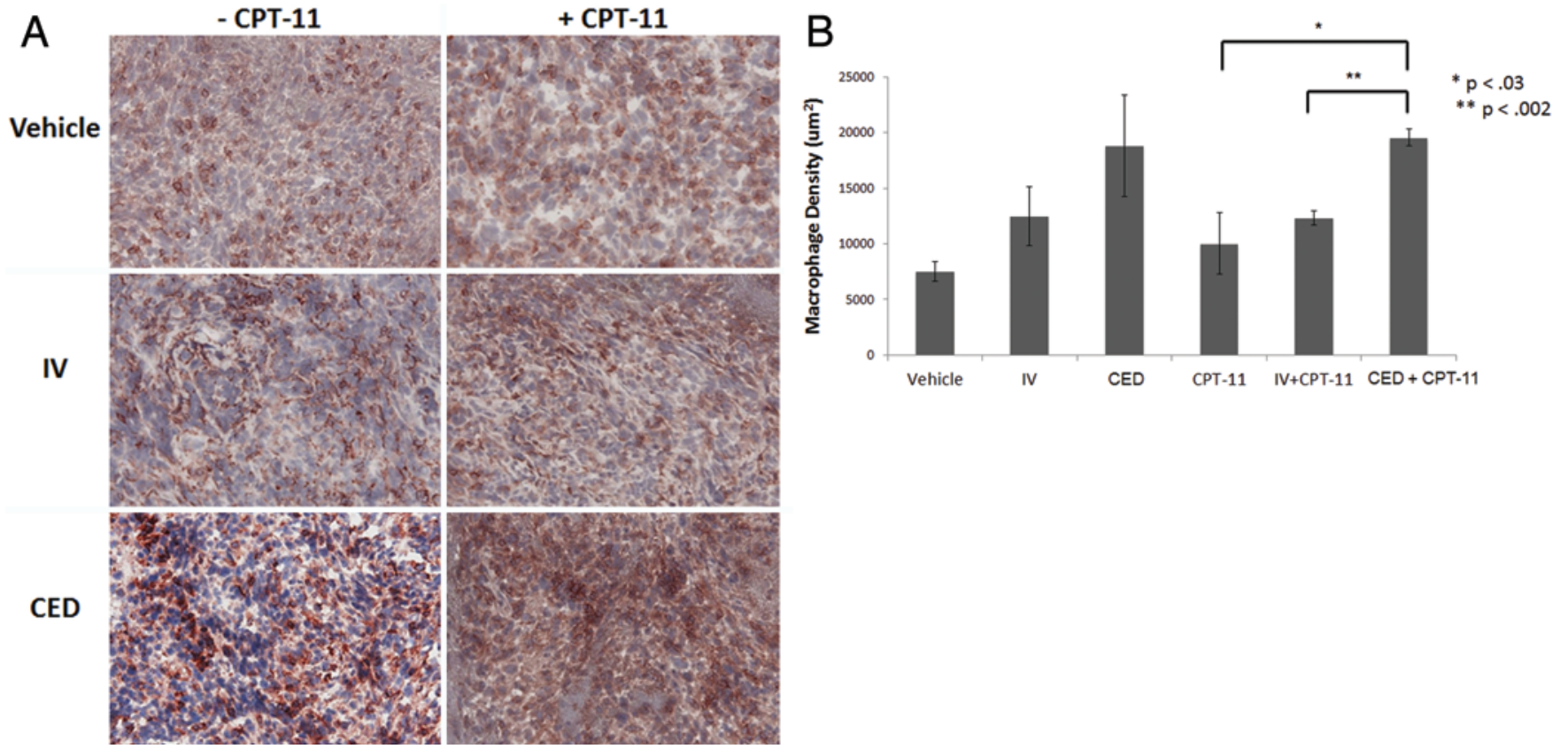

FIG. 4. Intratumoral administration of bevacizumab increased the numbers of macrophages in tumor tissues. Frozen sections of tumor tissues were immunostained for macrophages (F4/80). Red precipitation indicates positive staining. A: Tissues derived from animals treated with CED bevacizumab alone or in combination with CPT-11 show greater numbers of positive cells within the tumors than those from the animals treated with IV bevacizumab. Original magnification $\times 200$. B: ImageJ analysis of these tissues shows that macrophage density in tissue from animals treated with CED bevacizumab and CPT- 11 exhibits higher numbers of positive cells than those from animals treated with IV bevacizumab plus CPT-11 ( $n=2$ each for the vehicle and CPT-11 groups; $\mathrm{n}=3$ each for the IV bevacizumab, CED bevacizumab, IV bevacizumab plus CPT-11, and CED bevacizumab plus CPT-11 treatment groups).

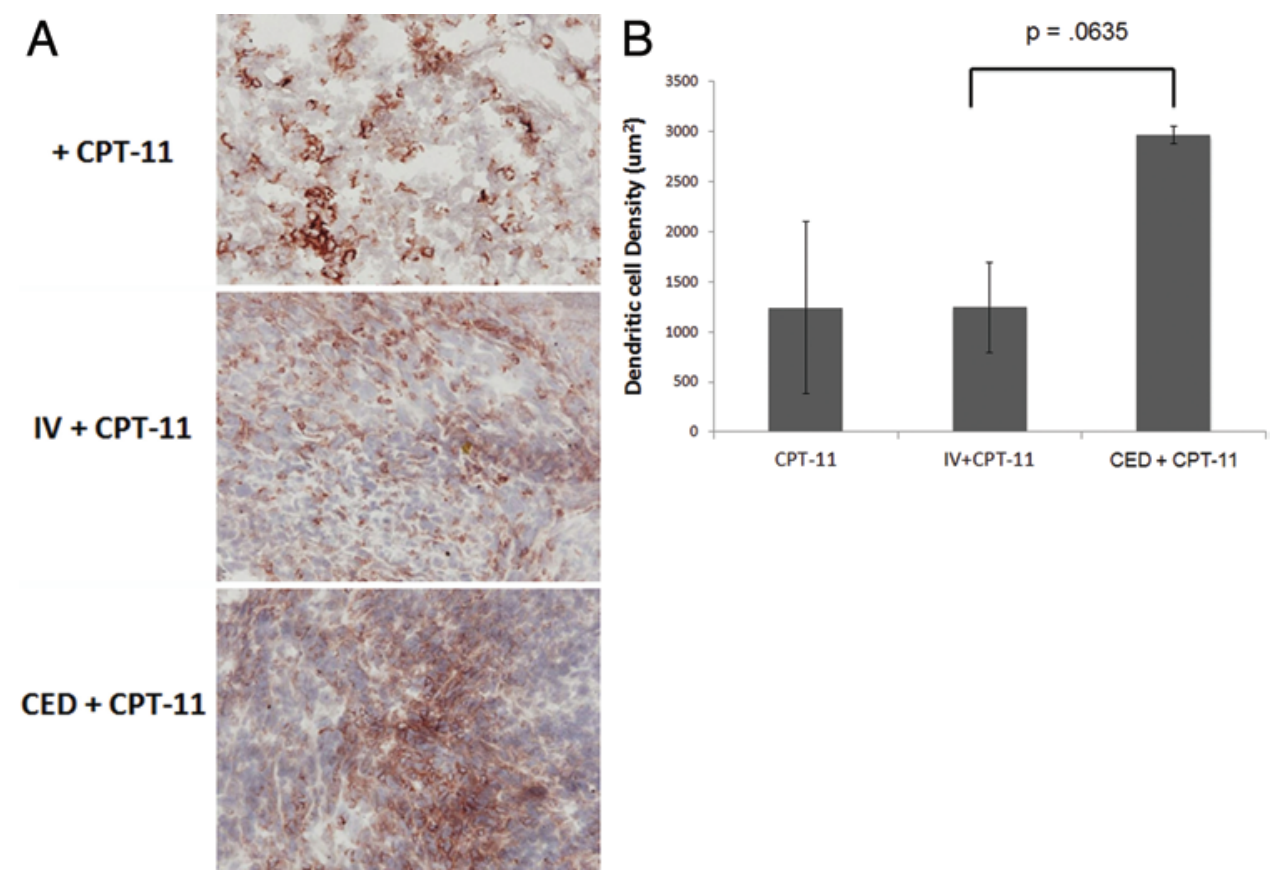

FIG. 5. CED of bevacizumab increased the numbers of dendritic cells in tumor tissues. Tumor tissue sections were immunostained with the dendritic cell marker CD11c. A: Tissue sections exhibit high numbers of dendritic cells throughout the tumor. Original magnification $\times 200$. B: Quantitation of positive cells shows an increased trend in the number of dendritic cells in animals treated with CED bevacizumab plus CPT-11 compared with that in animals treated with CPT-11 alone or CPT-11 plus IV bevacizumab. These groups were not significantly different ( $n=2$ for all groups). 
not accessed because staining with anti-VEGF produced high background staining (data not shown).

Clinical evidence has shown that halting bevacizumab treatment for more than 2 weeks causes a resurgence in tumor growth and extensive blood vessel regrowth within the tumor. 22,35 This tumor expansion has been attributed to several factors. Blocking VEGF activity has been shown to increase tumor cell invasion into the normal brain. ${ }^{20}$ Enhanced invasion is the result of the upregulation of enzymes responsible for the breakdown of extracellular matrix, such as MMP2, MMP9, and TIMP- $1 .{ }^{20}$ Enhanced invasion of tumor cells has also been attributed to migration into normal brain along the empty basement membrane sleeves left by the regressed blood vessels. ${ }^{1,22}$ Bevacizumab therapy has been associated with the gliomatosis-like diffuse and distant spread of recurring tumors. ${ }^{33}$ Our data with direct CED suggests that local delivery has less of an impact on gliomatosis. Thus, systemic administration may be effective in reducing tumor size but not in reducing tumor cell infiltration.

Systemic bevacizumab toxicity, including poor wound healing, hemorrhage, hypertension, proteinuria, bowel perforation, and fistula formation, is well known in clinical practice. However, these complications are often related to chronic bevacizumab infusion. Local delivery of bevacizumab also did not induce hemorrhage in the brain or in tumors and did not result in poor healing. We did not detect problems with hypertension, proteinuria, or bowel perforation in the mice treated with CED bevacizumab. A serious adverse effect of bevacizumab is an increased risk of thrombotic events. Our studies show that CED did not have this effect on the cerebral vasculature. Drugs administered through CED in human patients has been shown to be an effective way of achieving a high concentration of drug within the tumor. Because of the heterogeneity of the tumor itself, the vascularity of the tumor, and the differences in the blood-tumor interface, the ability to deliver bevacizumab to a glioblastoma intravenously is probably highly variable between the necrotic zones of the glioblastoma compared with the peripheral contrast-enhancing regions. Reported problems with anti-VEGF therapy may be remedied by changes in administration protocols, such as switching to CED. Unfortunately, patients develop nonresponsiveness to bevacizumab. ${ }^{26}$ The mechanism of nonresponsiveness is not well understood, but the current hypothesis is that with a constant decrease in VEGF in the tumor environment, other proangiogenic cytokines (e.g., fibroblast growth factor 2) are overexpressed, which leads to extensive resurgence of angiogenesis.

Although the results presented here are promising, there are still unresolved issues. First, IV bevacizumab has shown an impressive ability to decrease blood-tumor permeability, which leads to decreased enhancement with gadolinium, often referred to as a "super steroid." The degree by which CED of bevacizumab (versus IV bevacizumab) is able to decrease the blood-tumor barrier is not known. Unfortunately, we were unable to perform MRI scans of these rodent brains with or without gadolinium. Second, the CED/infusion schedule still needs to be determined. The Alzet pump allows for continuous osmotic delivery of bevacizumab into the tumor over a 28-day pe- riod. How the delivery protocol can be optimized as either a continuous or a pulsed delivery still needs to be determined. Moreover, the current limitations of CED (e.g., a catheter can be left in for only a certain period of time) are rate-limiting factors to this delivery system. Recently, an implanted pump that may be used for CED of drugs has been described. ${ }^{32}$

\section{Conclusions}

In summary, the results of our studies show that CED of bevacizumab with or without irinotecan results in increased survival, decreased tumor blood vessels, and increased inflammatory cell accumulation in the tumors. Although we did not directly compare the adverse effects of bevacizumab given systemically (IV) with those of it given by CED, systemic adverse effects such as poor wound healing, hypertension, renal insufficiency, and deep venous thrombosis would be expected to decrease significantly after local CED. Further clinical studies using direct CED of bevacizumab in combination with cytotoxic chemotherapy should be considered for patients who have tumor progression while on high-dose temozolomide. Whether CED of bevacizumab can be used in long-term therapy may soon be addressed by the use of implantable programmable pumps currently in development. ${ }^{5}$ Thus, the positive effects of bevacizumab on vessel reduction make CED of bevacizumab a more efficient and effective means of therapy.

\section{Acknowledgments}

The authors thank Michael Weintraub and Bryna C. Tsai for their expert help with the in vivo experiments.

\section{References}

1. Baffert F, Le T, Sennino B, Thurston G, Kuo CJ, Hu-Lowe $\mathrm{D}$, et al: Cellular changes in normal blood capillaries undergoing regression after inhibition of VEGF signaling. Am J Physiol Heart Circ Physiol 290:H547-H559, 2006

2. Barbera-Guillem E, Nyhus JK, Wolford CC, Friece CR, Sampsel JW: Vascular endothelial growth factor secretion by tumor-infiltrating macrophages essentially supports tumor angiogenesis, and IgG immune complexes potentiate the process. Cancer Res 62:7042-7049, 2002

3. Calabrese C, Poppleton H, Kocak M, Hogg TL, Fuller C, Hamner B, et al: A perivascular niche for brain tumor stem cells. Cancer Cell 11:69-82, 2007

4. Carmeliet P, Jain RK: Angiogenesis in cancer and other diseases. Nature 407:249-257, 2000

5. Chen TC, Napolitano GR, Adell F, Schönthal AH, Shachar Y: Development of medtronomic biofeedback pump for leptomeningeal carcinomatosis: technical note. J Neurosurg (in press), 2014

6. Chowdhary S, Chamberlain M: Bevacizumab for the treatment of glioblastoma. Expert Rev Neurother 13:937-949, 2013

7. Desjardins A, Reardon DA, Herndon JE II, Marcello J, Quinn JA, Rich JN, et al: Bevacizumab plus irinotecan in recurrent WHO grade 3 malignant gliomas. Clin Cancer Res 14:7068-7073, 2008

8. Dvorak HF: Vascular permeability factor/vascular endothelial growth factor: a critical cytokine in tumor angiogenesis and a potential target for diagnosis and therapy. J Clin Oncol 20:4368-4380, 2002 
9. Everson RG, Graner MW, Gromeier M, Vredenburgh JJ, Desjardins A, Reardon DA, et al: Immunotherapy against angiogenesis-associated targets: evidence and implications for the treatment of malignant glioma. Expert Rev Anticancer Ther 8:717-732, 2008

10. Ferrara N: Role of vascular endothelial growth factor in regulation of physiological angiogenesis. Am J Physiol Cell Physiol 280:C1358-C1366, 2001

11. Folkins C, Shaked Y, Man S, Tang T, Lee CR, Zhu Z, et al: Glioma tumor stem-like cells promote tumor angiogenesis and vasculogenesis via vascular endothelial growth factor and stromal-derived factor 1. Cancer Res 69:7243-7251, 2009

12. Friedman HS, Prados MD, Wen PY, Mikkelsen T, Schiff D, Abrey LE, et al: Bevacizumab alone and in combination with irinotecan in recurrent glioblastoma. J Clin Oncol 27:47334740, 2009

13. Goel HL, Mercurio AM: VEGF targets the tumour cell. Nat Rev Cancer 13:871-882, 2013

14. Goldszmid RS, Dzutsev A, Trinchieri G: Host immune response to infection and cancer: unexpected commonalities. Cell Host Microbe 15:295-305, 2014

15. Hein M, Graver S: Tumor cell response to bevacizumab single agent therapy in vitro. Cancer Cell Int 13:94, 2013

16. Hoeben A, Landuyt B, Highley MS, Wildiers H, Van Oosterom AT, De Bruijn EA: Vascular endothelial growth factor and angiogenesis. Pharmacol Rev 56:549-580, 2004

17. Iwamoto FM, Abrey LE, Beal K, Gutin PH, Rosenblum MK, Reuter VE, et al: Patterns of relapse and prognosis after bevacizumab failure in recurrent glioblastoma. Neurology 73: 1200-1206, 2009

18. Jain RK, di Tomaso E, Duda DG, Loeffler JS, Sorensen AG, Batchelor TT: Angiogenesis in brain tumours. Nat Rev Neurosci 8:610-622, 2007

19. Kreisl TN, Kim L, Moore K, Duic P, Royce C, Stroud I, et al: Phase II trial of single-agent bevacizumab followed by bevacizumab plus irinotecan at tumor progression in recurrent glioblastoma. J Clin Oncol 27:740-745, 2009

20. Lucio-Eterovic AK, Piao Y, de Groot JF: Mediators of glioblastoma resistance and invasion during antivascular endothelial growth factor therapy. Clin Cancer Res 15:45894599, 2009

21. Ma J, Waxman DJ: Combination of antiangiogenesis with chemotherapy for more effective cancer treatment. Mol Cancer Ther 7:3670-3684, 2008

22. Mancuso MR, Davis R, Norberg SM, O'Brien S, Sennino B, Nakahara T, et al: Rapid vascular regrowth in tumors after reversal of VEGF inhibition. J Clin Invest 116:2610-2621, 2006

23. Merad M, Sathe P, Helft J, Miller J, Mortha A: The dendritic cell lineage: ontogeny and function of dendritic cells and their subsets in the steady state and the inflamed setting. Annu Rev Immunol 31:563-604, 2013

24. Nghiemphu PL, Liu W, Lee Y, Than T, Graham C, Lai A, et al: Bevacizumab and chemotherapy for recurrent glioblastoma: a single-institution experience. Neurology 72:1217-1222, 2009

25. Plate KH, Breier G, Weich HA, Mennel HD, Risau W:
Vascular endothelial growth factor and glioma angiogenesis: coordinate induction of VEGF receptors, distribution of VEGF protein and possible in vivo regulatory mechanisms. Int J Cancer 59:520-529, 1994

26. Rahman R, Smith S, Rahman C, Grundy R: Antiangiogenic therapy and mechanisms of tumor resistance in malignant glioma. J Oncol 2010:251231, 2010

27. Rahmathulla G, Hovey EJ, Hashemi-Sadraei N, Ahluwalia MS: Bevacizumab in high-grade gliomas: a review of its uses, toxicity assessment, and future treatment challenges. Onco Targets Ther 6:371-389, 2013

28. Simon T, Coquerel B, Petit A, Kassim Y, Demange E, Le Cerf D, et al: Direct effect of bevacizumab on glioblastoma cell lines in vitro. Neuromolecular Med 16:752-771, 2014

29. Soltau J, Drevs J: Mode of action and clinical impact of VEGF signaling inhibitors. Expert Rev Anticancer Ther 9:649-662, 2009

30. Stupp R, Hegi ME, Gilbert MR, Chakravarti A: Chemoradiotherapy in malignant glioma: standard of care and future directions. J Clin Oncol 25:4127-4136, 2007

31. von Baumgarten L, Brucker D, Tirniceru A, Kienast Y, Grau S, Burgold S, et al: Bevacizumab has differential and dosedependent effects on glioma blood vessels and tumor cells. Clin Cancer Res 17:6192-6205, 2011

32. Wang W, Ghandi A, Liebes L, Louie SG, Hofman FM, Schönthal AH, et al: Effective conversion of irinotecan to SN-38 after intratumoral drug delivery to an intracranial murine glioma model in vivo. Laboratory investigation. J Neurosurg 114:689-694, 2011

33. Wick W, Wick A, Weiler M, Weller M: Patterns of progression in malignant glioma following anti-VEGF therapy: perceptions and evidence. Curr Neurol Neurosci Rep 11:305-312, 2011

34. Ziegler-Heitbrock L, Ancuta P, Crowe S, Dalod M, Grau V, Hart DN, et al: Nomenclature of monocytes and dendritic cells in blood. Blood 116:e74-e80, 2010

35. Zuniga RM, Torcuator R, Jain R, Anderson J, Doyle T, Ellika $\mathrm{S}$, et al: Efficacy, safety and patterns of response and recurrence in patients with recurrent high-grade gliomas treated with bevacizumab plus irinotecan. J Neurooncol 91:329_ 336,2009

\section{Author Contributions}

Conception and design: Hofman, Sivakumar, Schönthal, Chen. Acquisition of data: Wang, Sivakumar, Torres, Vaikari, Gong, Howard. Analysis and interpretation of data: Hofman, Jhaveri, Gong, Howard, Golden, Louie, Schönthal, Chen. Drafting the article: Hofman, Wang, Jhaveri. Critically revising the article: Hofman, Jhaveri, Chen. Reviewed submitted version of manuscript: Hofman. Approved the final version of the manuscript on behalf of all authors: Hofman. Administrative/technical/material support: Chen. Study supervision: Chen, Hofman.

\section{Correspondence}

Thomas C. Chen, Department of Neurosurgery, University of Southern California, 2011 Zonal Ave., Los Angeles, CA 90033. email: tchen68670@gmail.com. 